\title{
Enantioselective Synthesis of Four Stereoisomers of Sulfinyl Ferrocenyl Quinones with Central, Planar and Helical Chirality
}

\author{
Ana M. del Hoyo, Antonio Urbano,* M. Carmen Carreño*
}

carmen.carrenno@uam.es, antonio.urbano@uam.es

\section{Experimental Procedures}

General. Melting points were obtained in open capillary tubes and are uncorrected. ${ }^{1} \mathrm{H}$ - and ${ }^{13} \mathrm{C}-\mathrm{NMR}$ spectra were recorded in $\mathrm{CDCl}_{3}$ at 300 and $75 \mathrm{MHz}$, respectively. All reactions were monitored by thin layer chromatography that was performed on precoated sheets of silica gel 60, and flash column chromatography was done with silica gel 60 (230-400 mesh) of Merck. Eluting solvents are indicated in the text. The apparatus for inert atmosphere experiments was dried by flaming in a stream of dry nitrogen. Ethyl ether, $\mathrm{CH}_{2} \mathrm{Cl}_{2}$ and THF were dried over $4 \AA$ molecular sieves. All other reagent quality solvents were used without purification. For routine workup, extraction was carried out with ethyl acetate, and solvent drying with $\mathrm{MgSO}_{4}$.

Measurements. UV/spectra were recorded in on a JASCO V-660 spectrophotometer and circular dichroism spectra were measured with JASCO J-815 CD spectropolarimeter. The spectra were measured in $\mathrm{CH}_{2} \mathrm{Cl}_{2}$ in a quarzt cuvette $(1 \mathrm{~cm})$ at $298 \mathrm{~K}$. 


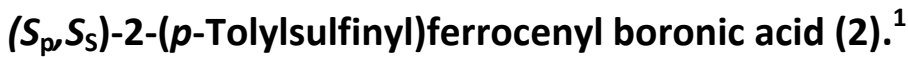

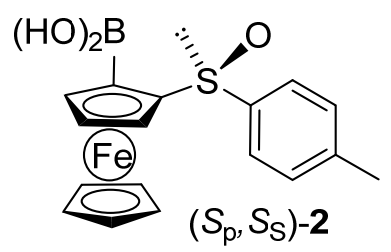

To $\left(S_{\mathrm{S}}\right)$-ferrocenyl $p$-tolylsulfoxide ${ }^{2}(4.1 \mathrm{~g}, 12.6 \mathrm{mmol})$ in THF $(130 \mathrm{~mL})$ at $-78{ }^{\circ} \mathrm{C}$ under argon, LDA, prepared from $n$-BuLi $2.4 \mathrm{M}$ in hexanes $(5.6 \mathrm{~mL}, 13.9 \mathrm{mmol})$, diisopropylamine $(1.96 \mathrm{~mL}, 13.9 \mathrm{mmol})$ and THF $(6.4 \mathrm{~mL})$, was added dropwise via cannula. The resulting orange-red solution was stirred at $-78^{\circ} \mathrm{C}$ for 30 min before adding $\mathrm{B}(\mathrm{OMe})_{3}(4.4 \mathrm{~mL}, 39.2 \mathrm{mmol})$ and after stirring $30 \mathrm{~min}$, an additional amount of $\mathrm{B}(\mathrm{OMe})_{3}(4.4 \mathrm{~mL}, 39.2 \mathrm{mmol})$ was added. After 1 hour at the same temperature, the resulting yellow solution was slowly warmed to room temperature and then saturated $\mathrm{NH}_{4} \mathrm{Cl}(\mathrm{aq})$ was added. The aqueous phase was extracted with ethyl ether and dried over $\mathrm{MgSO}_{4}$. Concentration in vacuo afforded pure $\left(S_{\mathrm{p}}, S\right)-\mathbf{2}$, as a yellow solid, in quantitative yield $(4.6 \mathrm{~g})$.

$[\alpha]_{20}^{\mathrm{D}}=+492\left(c=0.50, \mathrm{CHCl}_{3}\right)$.

${ }^{1} \mathrm{H}$ NMR $\delta\left(300 \mathrm{MHz}, \mathrm{CDCl}_{3}\right): 7.38$ and 7.19 (AA'BB' system, $\left.J=8.5 \mathrm{~Hz}, 4 \mathrm{H}\right), 6.92$ (bs, $2 \mathrm{H}), 4.84(\mathrm{~m}, 1 \mathrm{H}), 4.69(\mathrm{~m}, 1 \mathrm{H}), 4.62(\mathrm{~m}, 1 \mathrm{H}), 4.46(\mathrm{~s}, 5 \mathrm{H}), 2.35(\mathrm{~s}, 3 \mathrm{H})$.

\section{$\left(R_{\mathrm{p}}, R_{\mathrm{S}}\right)$-2-(p-Tolylsulfinyl)ferrocenyl boronic acid (2).}

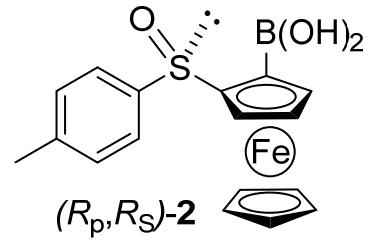

Compound $\left(R_{\mathrm{p}}, R\right)-\mathbf{2}$ was obtained from $(R)$-ferrocenyl $p$-tolyl sulfoxide ${ }^{2}$ following the same procedure.

$[\alpha]_{\mathrm{D}}^{20}=-495\left(c=0.50, \mathrm{CHCl}_{3}\right)$.

\footnotetext{
1 J. G. Seitzberg, C. Dissing, I. Sotofte, P. O. Norrby, M. Johannsen, J. Org. Chem. 2005, 70, 8332.

2 O. Riant, G. Argouarch, D. Guillaneux, O. Samuel, H. B. Kagan, J. Org. Chem. 1998, 63, 3511.
} 


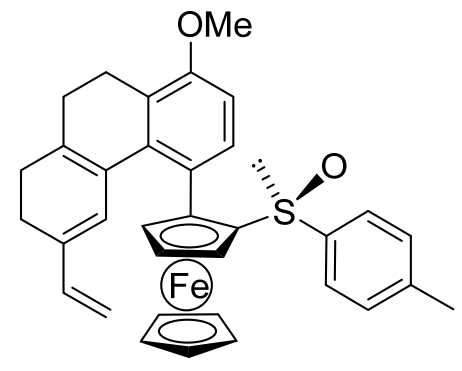

$\left(S_{p}, S_{S}\right)-3$

To a mixture of bromo tetrahydrophenanthrene $1^{3}$ (139 mg, $\left.0.438 \mathrm{mmol}\right),\left(S_{p}, S_{S}\right)-2-(p$ tolylsulfinyl)ferrocenyl boronic acid $2\left(403 \mathrm{mg}, 1.09 \mathrm{mmol}\right.$ ) and $\mathrm{Pd}(\mathrm{dppf}) \mathrm{Cl}_{2}(46 \mathrm{mg}$, $0.063 \mathrm{mmol})$, under nitrogen, toluene $(10 \mathrm{~mL})$ and $\mathrm{NaOH}(\mathrm{aq})(2.3 \mathrm{~mL}, 3 \mathrm{M})$ were added. The resulting suspension was heated at $110{ }^{\circ} \mathrm{C}$ for $2 \mathrm{~h}$, extracted with EtOAC and concentrated in vacuo. The crude reaction mixture was purified by flash chromatography (hexane/EtOAc $5: 1$ in $\mathrm{Al}_{2} \mathrm{O}_{3}$ deactivated with $10 \%$ of water), yielding a $55 \%$ of $\left(S_{p}, S_{s}\right)-3(135 \mathrm{mg})$, as a yellow oil.

$[\alpha]_{D}^{20}=+42\left(c 0.057, \mathrm{CH}_{2} \mathrm{Cl}_{2}\right), 98 \%$ ee.

HPLC: Daicel Chiralpak IB, hexane / 2-propanol 85:15; $0.7 \mathrm{~mL} \mathrm{~min}^{-1}, 254 \mathrm{~nm}, R_{t}=30,1$ $\min , T=25 \stackrel{\circ}{ } \mathrm{C})$.

${ }^{1} \mathrm{H}$ NMR $\delta\left(300 \mathrm{MHz}, \mathrm{CDCl}_{3}\right): \delta 8.06$ and $6.88(\mathrm{AB}$ system, $J=8.6 \mathrm{~Hz}, 2 \mathrm{H}), 7.61$ and 7.25 (AA'BB' system, $J=8.6 \mathrm{~Hz}, 4 \mathrm{H}), 5.94(\mathrm{dd}, J=17.5,10.7 \mathrm{~Hz}, 1 \mathrm{H}), 5.04-4.90(\mathrm{~m}, 2 \mathrm{H})$, $4.80(\mathrm{~d}, J=10.8 \mathrm{~Hz}, 1 \mathrm{H}), 4.28(\mathrm{~s}, 1 \mathrm{H}), 4.21(\mathrm{~s}, 1 \mathrm{H}), 4.13(\mathrm{bs}, 6 \mathrm{H}), 3.90(\mathrm{~s}, 3 \mathrm{H}), 3.30-2.86$ $(\mathrm{m}, 1 \mathrm{H}), 2.37(\mathrm{~s}, 4 \mathrm{H}), 2.30-2.09(\mathrm{~m}, 2 \mathrm{H}), 2.04(\mathrm{~s}, 1 \mathrm{H})$.

${ }^{13} \mathrm{C}$ NMR $\delta\left(75 \mathrm{MHz} \mathrm{CDCl}_{3}\right):$ 155.2, 141.0, 140.7, 139.0, 138.5, 137.0, 133.4, 130.0, $129.1,128.4,125.2,125.0,122.1$, 109.0, 108.0, 93.4, 91.8, 75.2, 70.8, 67.9, 67.4, 55.56, $29.2,27.8,27.7,27.0,21.4,21.2,21.2,13.6$.

MS (ESI+): $m / z(\%) 561\left(\mathrm{M}^{+}+1,40\right), 422(100)$.

HRMS: Calculated for $\mathrm{C}_{34} \mathrm{H}_{32} \mathrm{FeO}_{2} \mathrm{~S}\left(\mathrm{M}^{+}\right)$560.1545, found 560.1552

\footnotetext{
3 A. Latorre, A. Urbano, M. C. Carreño, Chem. Commun. 2011, 47, 1283
} 
$\left(R_{p}, R_{S}\right)$-5-(1-p-Tolylsulfinyl-ferrocene)-8-methoxy-3-vinyl-1,2,9,10-

tetrahydrophenantrene (3).

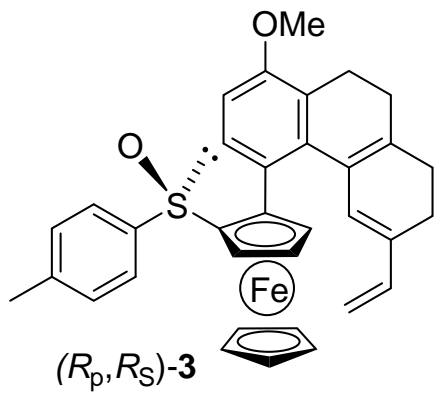

Compound $\left(R_{\mathrm{p}}, R_{\mathrm{S}}\right)$-3 was obtained from $\left(R_{\mathrm{p}}, R_{\mathrm{S}}\right)$-2-( $p$-tolylsulfinyl)ferrocenyl boronic acid (2) following the same procedure.

$[\alpha]_{D}^{20}=-80\left(c=0.034, \mathrm{CH}_{2} \mathrm{Cl}_{2}\right), 97 \%$ ee.

HPLC: Daicel Chiralpak IB, hexane / 2-propanol 85:15; $0.7 \mathrm{~mL} \mathrm{~min}^{-1}, 254 \mathrm{~nm}, R_{t}=13.6$ $\min , T=25 \stackrel{\circ}{ } \mathrm{C})$.

$\left(P, S_{p}, S_{S}\right)$-14-(1-p-Tolylsulfinyl-ferrocene)-11-methoxy-3-methyl-7,8,9,10-tetrahydro[5]-helicenequinone (5)

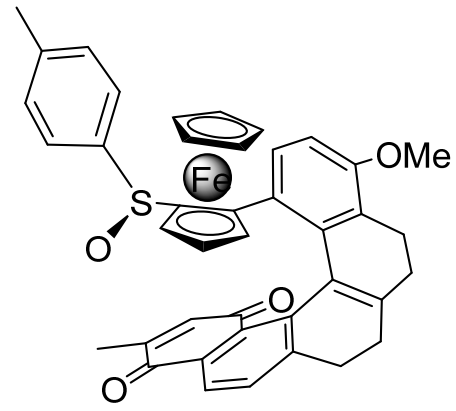

$\left(P, S_{\mathrm{p}}, S_{\mathrm{S}}\right)-5$

To a mixture of 3-vinyltetrahydrophenanthrene $\left(S_{p}, S_{s}\right)-3(62 \mathrm{mg}, 0.11 \mathrm{mmol})$ and $\left(S_{s}\right)-5$ methyl-2-(p-tolylsulfinyl)-1,4-benzoquinone (4) $(57 \mathrm{mg}, 0.22 \mathrm{mmol})$ at $-25{ }^{\circ} \mathrm{C}, \mathrm{CH}_{2} \mathrm{Cl}_{2}$ $(5.7 \mathrm{~mL})$ was rapidly added, under argon. After 15 hours, the reaction mixture was allowed to warm to room temperature and was stirred for 24 hours. After elimination of the solvent and flash chromatography (hexane/EtOAc 2:1), compound $\left(P, S_{p}, S_{S}\right)-5$ was obtained in $68 \%$ yield $(51 \mathrm{mg})$, as a brown solid. 
$[\alpha]_{D}{ }^{20}=+452\left(c \quad 0.042, \mathrm{CH}_{2} \mathrm{Cl}_{2}\right), 94 \%$ ee.

HPLC: Daicel Chiralpak IB, hexane / 2-propanol 85:15; $0.9 \mathrm{~mL} \mathrm{~min}^{-1}, 254 \mathrm{~nm}, R_{t}=75.1$ $\min , T=25 \stackrel{\circ}{\circ})$.

${ }^{1} \mathrm{H}$ NMR $\delta\left(300 \mathrm{MHz}, \mathrm{CDCl}_{3}\right): \delta$ 7.70-7.63 (AA'BB' system, $\left.J=7.9 \mathrm{~Hz}, 4 \mathrm{H}\right), 7.36(\mathrm{~d}, J=8.7$ $\mathrm{Hz}, 2 \mathrm{H}), 7.28(\mathrm{~d}, J=9.1 \mathrm{~Hz}, 1 \mathrm{H}), 6.86(\mathrm{~d}, J=8.7 \mathrm{~Hz}, 1 \mathrm{H}), 6.49(\mathrm{~d}, J=1.5 \mathrm{~Hz}, 1 \mathrm{H}), 3.96(\mathrm{~s}$, $3 \mathrm{H}), 3.80(\mathrm{~s}, 1 \mathrm{H}), 3.74(\mathrm{~s}, 6 \mathrm{H}), 3.53(\mathrm{~s}, 1 \mathrm{H}), 3.40(\mathrm{~d}, J=9.7 \mathrm{~Hz}, 1 \mathrm{H}), 2.71-2.51(\mathrm{~m}, 4 \mathrm{H})$, $2.44(\mathrm{~s}, 3 \mathrm{H}), 2.34(\mathrm{~m}, 2 \mathrm{H}), 2.06(\mathrm{~d}, J=1.5 \mathrm{~Hz}, 3 \mathrm{H}), 2.02(\mathrm{bs}, 1 \mathrm{H})$.

${ }^{13} \mathrm{C}$ NMR $\delta$ (75 MHz, CDCl $)$ ): 186.7, 184.6, 154.7, 147.8, 144.8, 143.9, 143.0, 141.5, 138.0, 137.6, 136.6, 132.4, 132.1, 131.9, 130.0, 129.7, 129.4, 127.2, 125.9, 125.9, 124.3, 120.4, 107.6, 94.1, 91.2, 71.1, 70.9, 66.7, 66.3, 53.4, 29.7, 29.2, 28.9, 21.5, 21.4, 16.0 .

MS (ESI+): $m / z$ (\%) $679\left(\mathbf{M}^{+}+1,100\right), 540$ (27).

HRMS: Calculated for $\mathrm{C}_{41} \mathrm{H}_{34} \mathrm{FeO}_{4} \mathrm{~S}\left(\mathrm{M}^{+}\right)$678.1527, found 678.1579.

14-(1-p-Tolylsulfinyl-ferrocenyl)-11-methoxy-3-methyl-5,7,8,9,10,15-hexahydro-[5]helicenequinone (6)

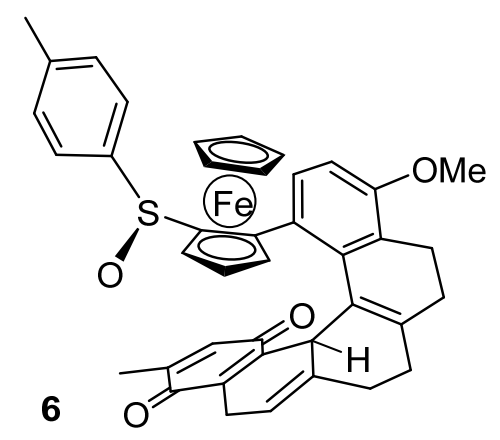

To a mixture of 3-vinyltetrahydrophenanthrene $\left(S_{p}, S_{S}\right)-3(65 \mathrm{mg}, 0.116 \mathrm{mmol})$ and $\left(S_{S}\right)$ 5-methyl-2-(p-tolylsulfinyl)-1,4-benzoquinone (4) (30 mg, $0.116 \mathrm{mmol})$ at $-25{ }^{\circ} \mathrm{C}$, $\mathrm{CH}_{2} \mathrm{Cl}_{2}(5.7 \mathrm{~mL})$ was rapidly added, under argon, and stirred for 15 hours at the same temperature. After elimination of the solvent and flash chromatography (hexane/EtOAc 2:1), compound 6 was obtained in $72 \%$ yield $(57 \mathrm{mg}$ ), as a very unstable red oil.

$[\alpha]_{D}^{20}=+286\left(c=0.028, \mathrm{CH}_{2} \mathrm{Cl}_{2}\right)$, 
${ }^{1} \mathrm{H}$ NMR $\delta\left(300 \mathrm{MHz} \mathrm{CDCl}_{3}\right): 7.59(\mathrm{~d}, J=8.4 \mathrm{~Hz}, 1 \mathrm{H}), 7.25-7.19(\mathrm{~m}, 4 \mathrm{H}), 6.74(\mathrm{~d}, J=8.5$ $\mathrm{Hz}, 1 \mathrm{H}), 6.13$ (bs, 1H), 5.54 (bs, 1H), 4.65 (s, 1H), 4.22 (s, 1H), 4.05 (s, 1H), 3.94 (s, 3H), $3.87(\mathrm{~s}, 5 \mathrm{H}), 3.46(\mathrm{dd}, J=19.1,5.4 \mathrm{~Hz}, 1 \mathrm{H}), 3.26(\mathrm{t}, J=13.9 \mathrm{~Hz}, 2 \mathrm{H}), 2.79-2.50(\mathrm{~m}, 2 \mathrm{H})$, $2.40(\mathrm{~s}, 3 \mathrm{H}), 2.29-2.13(\mathrm{~m}, 5 \mathrm{H})$.

$\left(M, S_{p}, S_{S}\right)$-14-(1-p-Tolylsulfinyl-ferrocenyl)-11-methoxy-3-methyl-7,8,9,10-tetrahydro[5]-helicenequinone (7).

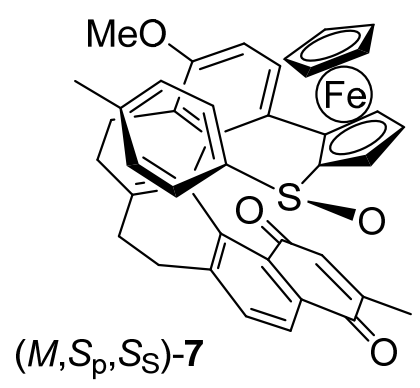

To a solution of diene $(\mathrm{rac})-3$ (39 mg, $0.07 \mathrm{mmol})$ in $\mathrm{CH}_{2} \mathrm{Cl}_{2}(1.8 \mathrm{~mL})$ at $-78{ }^{\circ} \mathrm{C}$, a solution of $\left(S_{S}\right)$-5-methyl-2-(p-tolylsulfinyl)-1,4-benzoquinone (4) $(8 \mathrm{mg}, 0.029 \mathrm{mmol}$, 0.41 equiv) in $\mathrm{CH}_{2} \mathrm{Cl}_{2}(0.2 \mathrm{M})$ was rapidly added under argon. After 1 hour at $-78^{\circ} \mathrm{C}$, the solvent was eliminated and the residue purified by flash chromatography (eluent hexane/EtOAc, from $3: 1$ to $1: 1$ ), to afford a $49 \%$ yield (19 $\mathrm{mg}$ ) of unreacted optically active diene $\left(R_{\mathrm{p}}, R_{\mathrm{S}}\right)-3\left\{[\alpha]_{\mathrm{D}}^{20}=-21\left(\mathrm{c} 0.051, \mathrm{CH}_{2} \mathrm{Cl}_{2}\right), 70 \%\right.$ ee $\}$, and a $25 \%$ yield $(12 \mathrm{mg})$ of ferrocenyl helicenequinone $\left(M, S_{p}, S_{S}\right)-7$, as a red solid.

$[\alpha]_{D}^{20}=-530\left(c 0.032, \mathrm{CH}_{2} \mathrm{Cl}_{2}\right), 97 \%$ ee.

HPLC: Daicel Chiralpak IB, hexane / 2-propanol 85:15; $0.7 \mathrm{~mL} \mathrm{~min}^{-1}, 254 \mathrm{~nm}, R_{t}=24.3$ $\min , T=25 \stackrel{\circ}{ } \mathrm{C})$.

${ }^{1} \mathrm{H}$ NMR $\delta\left(300 \mathrm{MHz} \mathrm{CDCl}_{3}\right): \delta 7.70$ and $7.36(\mathrm{AB}$ system, $J=7.7 \mathrm{~Hz}, 2 \mathrm{H}), 7.45$ and 6.80 ( $A B$ system, $J=8.6 \mathrm{~Hz}, 2 \mathrm{H}), 7.09$ and $6.87\left(\mathrm{AA}^{\prime} \mathrm{BB}^{\prime}\right.$ system, $\left.J=7.9 \mathrm{~Hz}, 4 \mathrm{H}\right), 6.46(\mathrm{~s}, 1 \mathrm{H})$, $4.30(\mathrm{~s}, 1 \mathrm{H}), 3.99(\mathrm{~s}, 3 \mathrm{H}), 3.93(\mathrm{~s}, 1 \mathrm{H}), 3.68(\mathrm{~s}, 1 \mathrm{H}), 3.61(\mathrm{~s}, 5 \mathrm{H}), 3.38$ (d, J = $14.1 \mathrm{~Hz}, 1 \mathrm{H})$, $3.27(\mathrm{dd}, J=15.5,8.8 \mathrm{~Hz}, 1 \mathrm{H}), 2.73-2.47(\mathrm{~m}, 6 \mathrm{H}), 2.32(\mathrm{~s}, 3 \mathrm{H}), 2.10(\mathrm{~s}, 3 \mathrm{H})$. 
${ }^{13} \mathrm{C}$ NMR $\delta\left(75 \mathrm{MHz} \mathrm{CDCl}_{3}\right): 186.1,184.5,155.4,149.2,146.4,145.1,144.5,140.4$, $137.6,136.6,134.8,132.7,131.3,131.1,129.7,128.8,128.5,126.4,124.4,124.4$, 120.1, 107.6, 99.2, 92.0, 73.1, 70.2, 67.3, 64.5, 53.4, 29.7, 29.4, 29.1, 28.8, 21.4, 21.3, 15.9.

MS (FAB+): $m / z(\%) 678\left(M^{+}, 100\right), 662(33)$.

HRMS: Calculated for $\mathrm{C}_{41} \mathrm{H}_{34} \mathrm{FeO}_{4} \mathrm{~S}\left(\mathrm{M}^{+}\right)$678.1527, found 678.1533 .

$\left(P, R_{p}, R_{s}\right)$-14-(1-p-Tolylsulfinyl-ferrocenyl)-11-methoxy-3-methyl-7,8,9,10-tetrahydro[5]-helicenequinone (7).

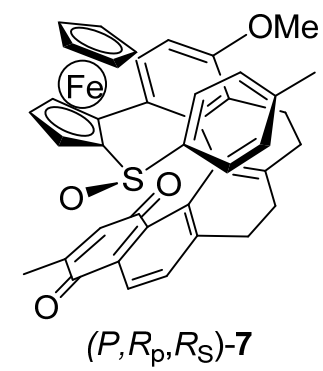

To a mixture of 3-vinyltetrahydrophenanthrene $\left(R_{\mathrm{p}}, R_{\mathrm{S}}\right)-3(62 \mathrm{mg}, 0.11 \mathrm{mmol})$ and (SS)5-methyl-2-(p-tolylsulfinyl)-1,4-benzoquinone (4) $\left(57 \mathrm{mg}, 0.22 \mathrm{mmol}\right.$ ) at $-25^{\circ} \mathrm{C}, \mathrm{CH}_{2} \mathrm{Cl}_{2}$ $(5.7 \mathrm{~mL})$ was rapidly added, under argon. After 24 hours, 2 equivalents of enantiopure sulfinyl quinone (57 $\mathrm{mg}, 0.22 \mathrm{mmol}$ ) were added, and the reaction mixture allowed to warm to room temperature and stirred for 5 days. After elimination of the solvent and flash chromatography (hexane/EtOAc 2:1), compound $\left(P, R_{\mathrm{p}}, R_{\mathrm{S}}\right)-7$ was obtained in $54 \%$ yield (40.3 mg), as a red solid.

$[\alpha]_{D}^{20}=+514\left(c 0.043, \mathrm{CH}_{2} \mathrm{Cl}_{2}\right),>98 \%$ ee.

HPLC: Daicel Chiralpak IB, hexane / 2-propanol 85:15; $0.7 \mathrm{~mL} \mathrm{~min}^{-1}, 254 \mathrm{~nm}, R_{t}=33.5$ $\min , T=25 \stackrel{\circ}{ } \mathrm{C})$.

${ }^{1} \mathbf{H}$ NMR $\delta\left(300 \mathrm{MHz} \mathrm{CDCl}_{3}\right): \delta 7.70$ and $7.36(\mathrm{AB}$ system, $J=7.7 \mathrm{~Hz}, 2 \mathrm{H}), 7.45$ and 6.80 ( $\mathrm{AB}$ system, $J=8.6 \mathrm{~Hz}, 2 \mathrm{H}), 7.09$ and $6.87\left(\mathrm{AA}^{\prime} \mathrm{BB}^{\prime}\right.$ system, $\left.J=7.9 \mathrm{~Hz}, 4 \mathrm{H}\right), 6.46(\mathrm{~s}, 1 \mathrm{H})$, 
$4.30(\mathrm{~s}, 1 \mathrm{H}), 3.99(\mathrm{~s}, 3 \mathrm{H}), 3.93(\mathrm{~s}, 1 \mathrm{H}), 3.68(\mathrm{~s}, 1 \mathrm{H}), 3.61(\mathrm{~s}, 5 \mathrm{H}), 3.38(\mathrm{~d}, J=14.1 \mathrm{~Hz}, 1 \mathrm{H})$, $3.27(\mathrm{dd}, J=15.5,8.8 \mathrm{~Hz}, 1 \mathrm{H}), 2.73-2.47(\mathrm{~m}, 6 \mathrm{H}), 2.32(\mathrm{~s}, 3 \mathrm{H}), 2.10(\mathrm{~s}, 3 \mathrm{H})$.

${ }^{13} \mathrm{C}$ NMR $\delta\left(75 \mathrm{MHz} \mathrm{CDCl}_{3}\right): 186.1,184.5,155.4,149.2,146.4,145.1,144.5,140.4$, 137.6, 136.6, 134.8, 132.7, 131.3, 131.1, 129.7, 128.8, 128.5, 126.4, 124.4, 124.4, 120.1, 107.6, 99.2, 92.0, 73.1, 70.2, 67.3, 64.5, 53.4, 29.7, 29.4, 29.1, 28.8, 21.4, 21.3, 15.9.

HRMS: Calculated for $\mathrm{C}_{41} \mathrm{H}_{34} \mathrm{FeO}_{4} \mathrm{~S}\left(\mathrm{M}^{+}\right)$678.1527, found 678.1513.

14-(1-p-Tolylsulfinyl-ferrocenyl)-11-methoxy-3-methyl-5,7,8,9,10,15-tetrahydro-[5]helicenequinone (8).

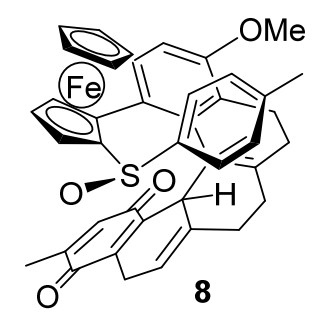

A mixture of 3-vinyltetrahydrophenanthrene $\left(R_{\mathrm{p}}, R_{\mathrm{S}}\right)-3(38 \mathrm{mg}, 0.068 \mathrm{mmol})$ and $\left(\mathrm{S}_{\mathrm{S}}\right)-5$ methyl-2-(p-tolylsulfinyl)-1,4-benzoquinone (4) (18 mg, $0.068 \mathrm{mmol})$ in $\mathrm{CH}_{2} \mathrm{Cl}_{2}$ (4.8 mL) was stirred at room temperature for $24 \mathrm{~h}$. After elimination of the solvent and flash chromatography (hexane/EtOAc 2:1), compound 8 was obtained in $60 \%$ yield $(27.7$ $\mathrm{mg}$ ), as a very unstable red oil.

$[\alpha]_{D}^{20}=+20\left(c 0.052, \mathrm{CH}_{2} \mathrm{Cl}_{2}\right)$

${ }^{1} \mathrm{H}$ NMR $\delta\left(300 \mathrm{MHz}, \mathrm{CDCl}_{3}\right): 7.80$ and 6.85 (AB system, $\left.J=8.5 \mathrm{~Hz} 1 \mathrm{H}\right), 7.58$ and 7.31 (AA'BB' system, $J=8.0 \mathrm{~Hz}, 4 \mathrm{H}), 6.09(\mathrm{~d}, J=1.4 \mathrm{~Hz}, 1 \mathrm{H}), 5.54(\mathrm{~s}, 1 \mathrm{H}), 4.41(\mathrm{~s}, 1 \mathrm{H}), 4-23(\mathrm{~s}$, 1H), $4.04(\mathrm{~s}, 5 \mathrm{H}), 3.93(\mathrm{~s}, 3 \mathrm{H}), 3.84(\mathrm{~s}, 1 \mathrm{H}), 3.54-3.38(\mathrm{~m}, 1 \mathrm{H}), 3.34-3.16(\mathrm{~m}, 2 \mathrm{H}), 2.75$ (td, $J=15.9,5.7 \mathrm{~Hz}, 1 \mathrm{H}), 2.42(\mathrm{~s}, 3 \mathrm{H}), 2.19(\mathrm{~m}, 6 \mathrm{H})$. 
[5]-helicenequinone (5).

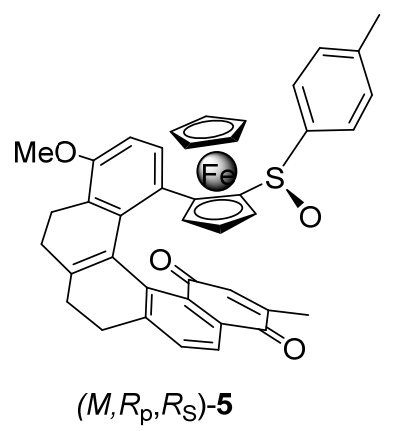

To a mixture of 3-vinyltetrahydrophenanthrene $\left(R_{\mathrm{p}}, R_{\mathrm{S}}\right)-3(27 \mathrm{mg}, 0.048 \mathrm{mmol})$ and (rac)-5-methyl-2-(p-tolylsulfinyl)-1,4-benzoquinone (4) (25 mg, $0.096 \mathrm{mmol}$ ) at $-78{ }^{\circ} \mathrm{C}$, $\mathrm{CH}_{2} \mathrm{Cl}_{2}(2.3 \mathrm{~mL})$ was rapidly added, under argon, and the reaction mixture was stirred for 5 hours. After elimination of the solvent and flash chromatography (hexane/EtOAc $2: 1)$, a $53 \%$ combined yield of a separable $50: 50$ mixture of compounds $\left(P, R_{p}, R_{S}\right)-7$ (8 $\mathrm{mg}, 88 \%$ ee) and $\left(M, R_{\mathrm{p}}, \mathrm{R}_{\mathrm{S}}\right)-\mathbf{5}(10 \mathrm{mg})$ was obtained.

$[\alpha]_{D}^{20}=-400\left(c \quad 0.02, \mathrm{CH}_{2} \mathrm{Cl}_{2}\right), 99 \%$ ee.

HPLC: Daicel Chiralpak IB, hexane / 2-propanol 85:15; $0.9 \mathrm{~mL} \mathrm{~min}^{-1}, 254 \mathrm{~nm}, R_{t}=34.7$ $\min , T=25 \stackrel{\circ}{ } \mathrm{C})$.

${ }^{1} \mathrm{H}$ NMR $\delta\left(300 \mathrm{MHz}, \mathrm{CDCl}_{3}\right)$ : $\delta$ 7.70-7.63 (AA'BB' system, $\left.J=7.9 \mathrm{~Hz}, 4 \mathrm{H}\right), 7.36(\mathrm{~d}, J=8.7$ $\mathrm{Hz}, 2 \mathrm{H}), 7.28(\mathrm{~d}, J=9.1 \mathrm{~Hz}, 1 \mathrm{H}), 6.86(\mathrm{~d}, J=8.7 \mathrm{~Hz}, 1 \mathrm{H}), 6.49(\mathrm{~d}, J=1.5 \mathrm{~Hz}, 1 \mathrm{H}), 3.96(\mathrm{~s}$, $3 \mathrm{H}), 3.80(\mathrm{~s}, 1 \mathrm{H}), 3.74(\mathrm{~s}, 6 \mathrm{H}), 3.53(\mathrm{~s}, 1 \mathrm{H}), 3.40(\mathrm{~d}, J=9.7 \mathrm{~Hz}, 1 \mathrm{H}), 2.71-2.51(\mathrm{~m}, 4 \mathrm{H})$, $2.44(\mathrm{~s}, 3 \mathrm{H}), 2.34(\mathrm{~m}, 2 \mathrm{H}), 2.06(\mathrm{~d}, J=1.5 \mathrm{~Hz}, 3 \mathrm{H}), 2.02(\mathrm{bs}, 1 \mathrm{H})$.

${ }^{13} \mathrm{C}$ NMR $\delta\left(75 \mathrm{MHz} \mathrm{CDCl}_{3}\right): 186.7,184.6,154.7,147.8,144.8,143.9,143.0,141.5$, $138.0,137.6,136.6,132.4,132.1,131.9,130.0,129.7,129.4,127.2$, 125.9, 125.9, 124.3, 120.4, 107.6, 94.1, 91.2, 71.1, 70.9, 66.7, 66.3, 53.4, 29.7, 29.2, 28.9, 21.5, 21.4, 16.0.

HRMS: Calculated for $\mathrm{C}_{41} \mathrm{H}_{34} \mathrm{FeO}_{4} \mathrm{~S}\left(\mathrm{M}^{+}\right)$678.1527, found 678.1582 . 


\section{$\underline{U V / V \text { data }}$}

\begin{tabular}{c|cc|} 
Band & $\mathbf{5}$ & $\mathbf{7}$ \\
& $\varepsilon\left(\mathrm{cm}^{-1} \mathrm{M}^{-1}\right) /[\mathrm{c}]=6.1 \cdot 10^{-5}$ & $\varepsilon\left(\mathrm{cm}^{-1} \mathrm{M}^{-1}\right) /[\mathrm{c}]=2.6 \cdot 10^{-4}$ \\
\hline $\mathbf{1}$ & $250 \mathrm{~nm}(14633)$ & $256 \mathrm{~nm}(22391)$ \\
2 & $465 \mathrm{~nm}(1551)$ & $465 \mathrm{~nm}(1534)$ \\
\cline { 2 - 3 }
\end{tabular}

\section{CD data}

\begin{tabular}{|c|c|c|c|c|}
\hline Band & $\begin{array}{c}\left(\boldsymbol{P}, \boldsymbol{S}_{P,}, \boldsymbol{S}_{S}\right)-5 \\
\Delta \varepsilon /[\mathrm{c}]=6.1 \cdot 10^{-5}\end{array}$ & $\begin{array}{c}\left(M, R_{P}, R_{S}\right)-5 \\
\Delta \varepsilon /[c]=1.5 \cdot 10^{-4}\end{array}$ & $\begin{array}{c}\left(\boldsymbol{P}, \boldsymbol{R}_{\boldsymbol{P}}, \boldsymbol{R}_{S}\right)-7 \\
\Delta \varepsilon /[\mathrm{c}]=2.6 \cdot 10^{-4}\end{array}$ & $\begin{array}{c}\left(M, S_{P}, S_{S}\right)-7 \\
\Delta \varepsilon /[c]=1.1 \cdot 10^{-4}\end{array}$ \\
\hline 1 & $\begin{array}{l}250 \mathrm{~nm}(-4.3), \\
278 \mathrm{~nm}(-2.0)\end{array}$ & $\begin{array}{l}253 \mathrm{~nm}(+8.1), \\
276 \mathrm{~nm}(+3.8)\end{array}$ & $284 \mathrm{~nm}(+0.57)$ & $287 \mathrm{~nm}(-2.8)$ \\
\hline 2 & $301 \mathrm{~nm}(+3.7)$ & 301 nm (-4.2) & $332 \mathrm{~nm}(+3.25)$ & 330 nm (-4.4) \\
\hline 3 & $384 \mathrm{~nm}(-1.1)$ & $388 \mathrm{~nm}(+1.2)$ & $427 \mathrm{~nm}(-0.6)$ & $424 \mathrm{~nm}(+1.4)$ \\
\hline 4 & $470 \mathrm{~nm}(+3.2)$ & $469 \mathrm{~nm}(-3.9)$ & $477 \mathrm{~nm}(+3.9)$ & $479 \mathrm{~nm}(-4.3)$ \\
\hline
\end{tabular}


HPLC chromatogram of (rac)-3

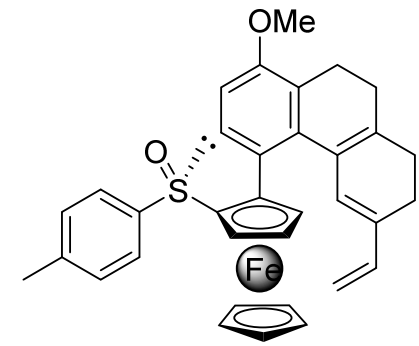

$\left(R_{\mathrm{p}}, R_{\mathrm{S}}\right)-3$

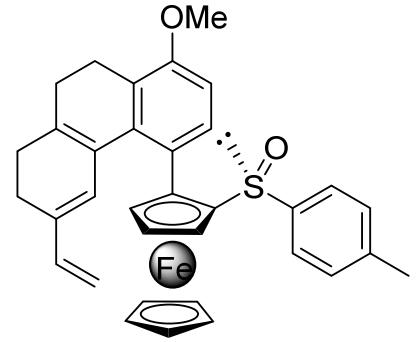

$\left(S_{p}, S_{S}\right)-3$
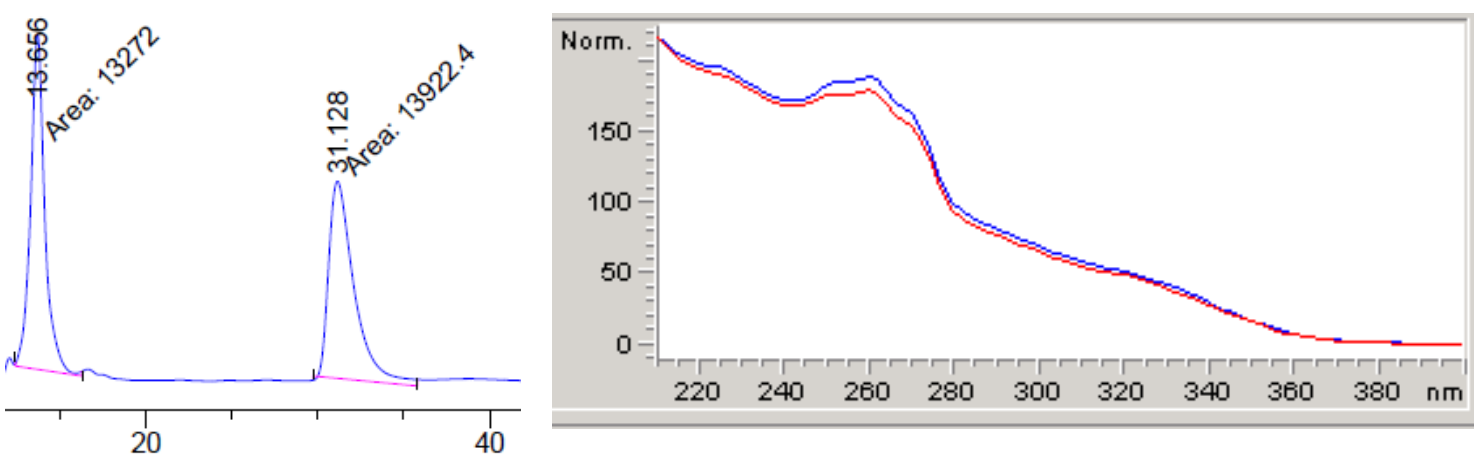

HPLC chromatogram of $\left(S_{p}, S_{S}\right)-3$
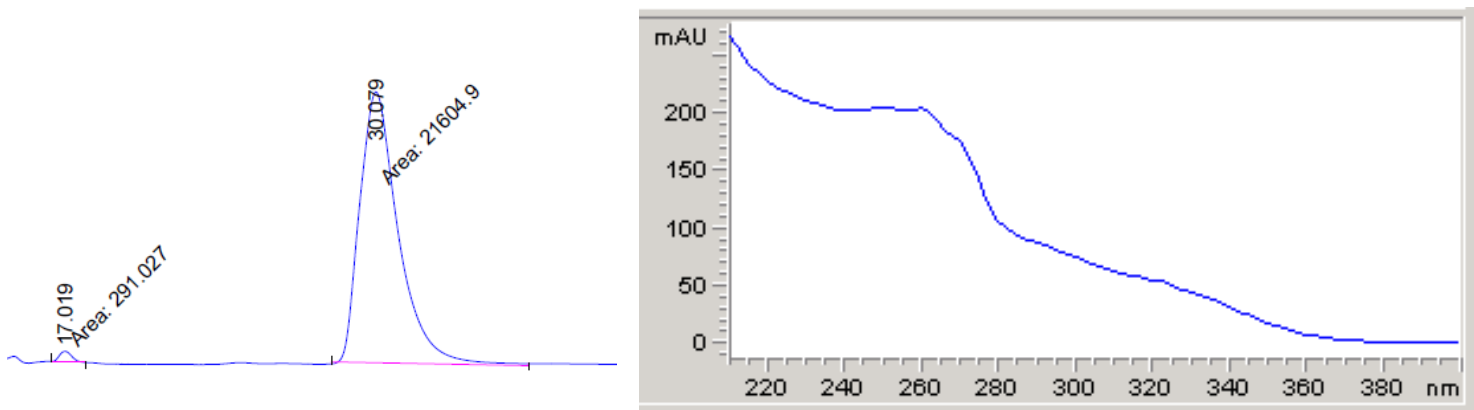

HPLC chromatogram of $\left(R_{\mathrm{p}}, R_{\mathrm{S}}\right)-\mathbf{3}$
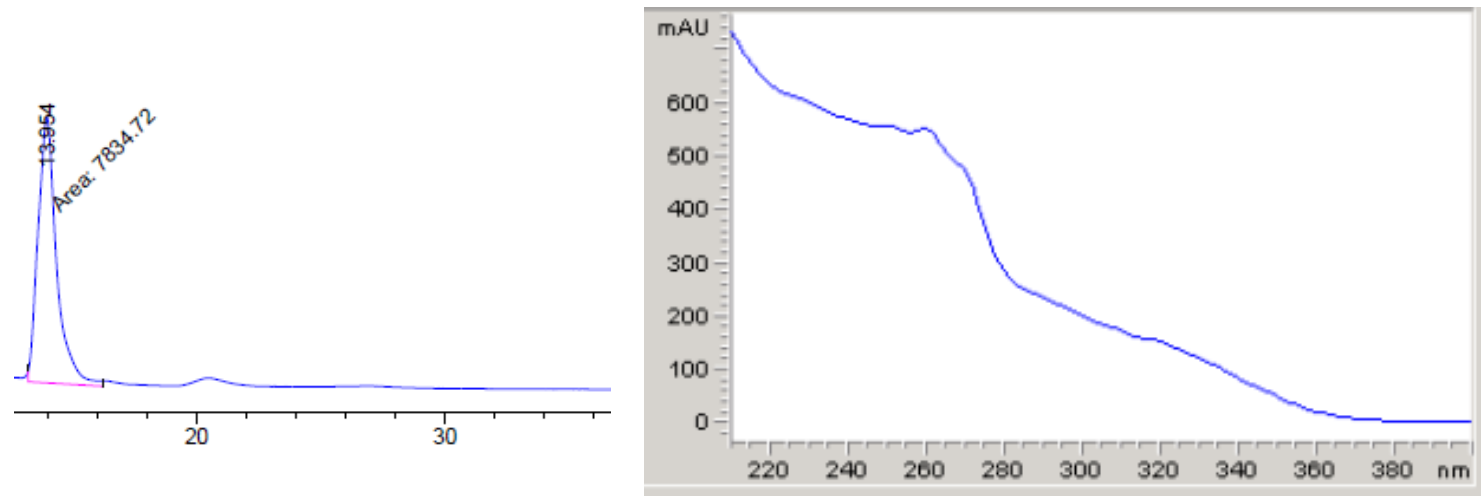
HPLC chromatogram of (rac)-5

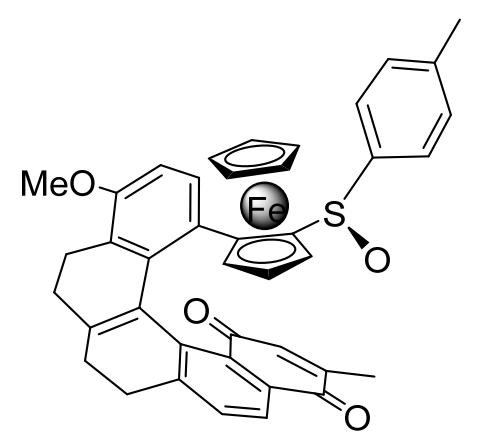

$\left(M, R_{\mathrm{p}}, R_{\mathrm{S}}\right)-5$

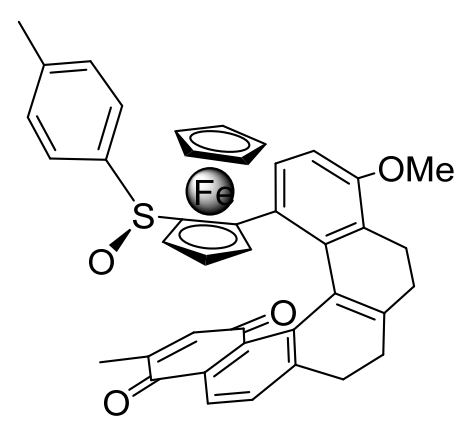

$\left(P, S_{p}, S_{S}\right)-5$
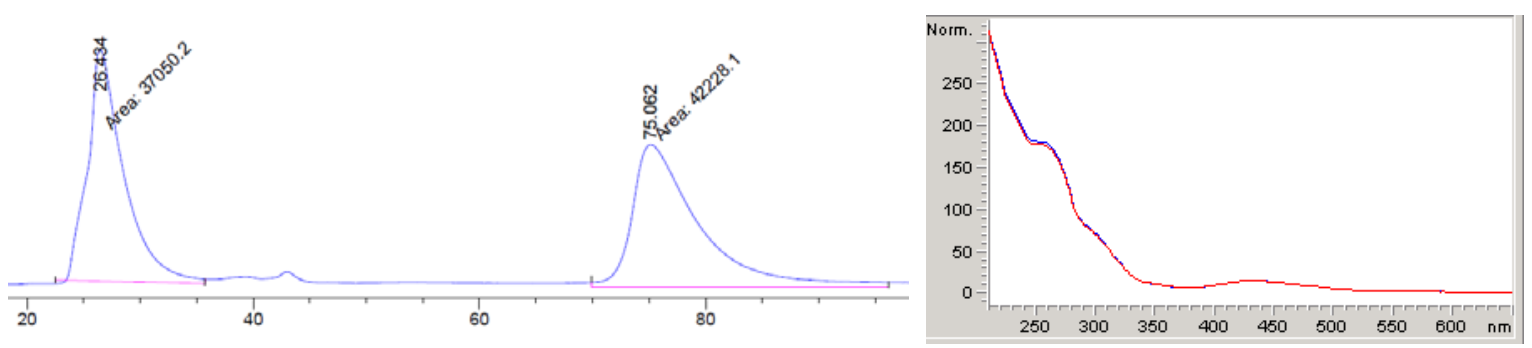

HPLC chromatogram of $\left(P, S_{p}, S_{S}\right)-5$

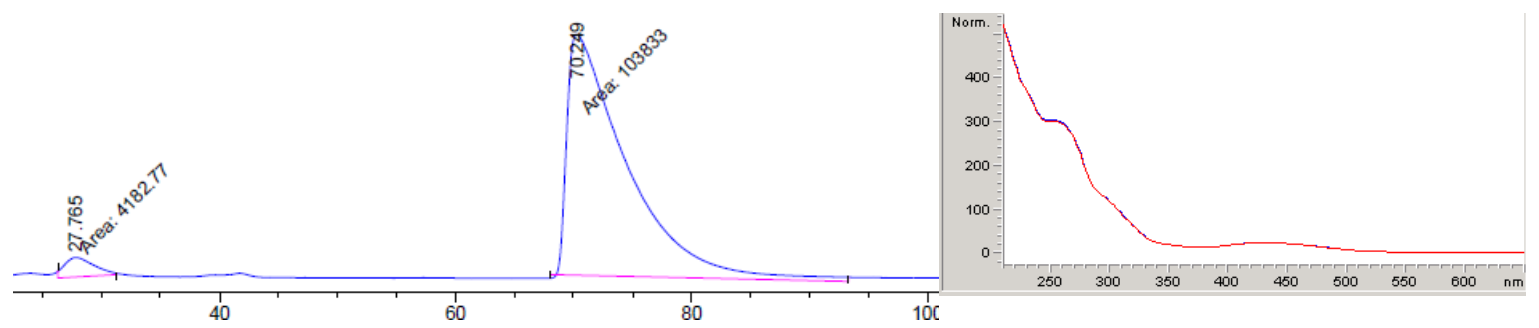

HPLC chromatogram of $\left(M, R_{\mathrm{p}}, R_{\mathrm{S}}\right)-5$
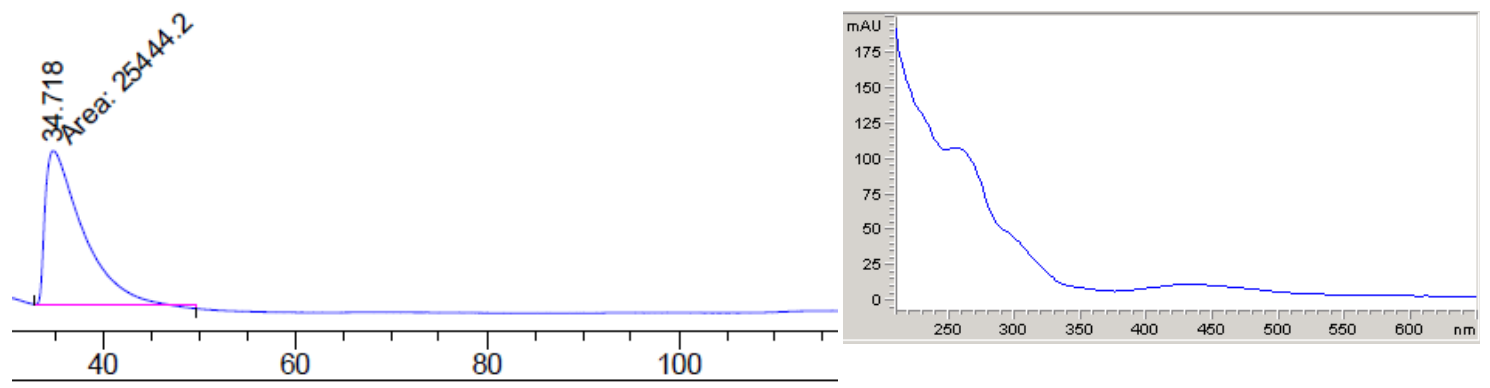
HPLC chromatogram of (rac)-7

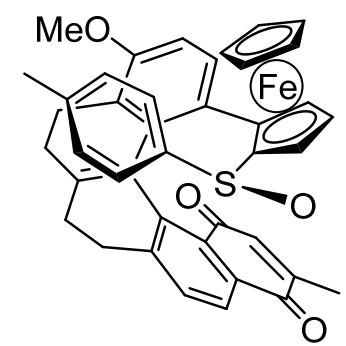

$\left(M, S_{p}, S_{S}\right)-7$

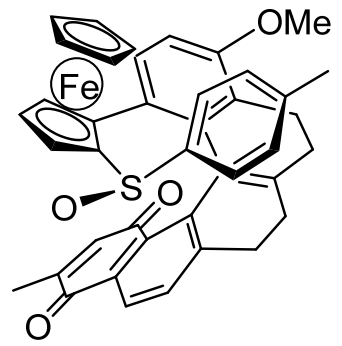

$\left(P, R_{\mathrm{p}}, R_{\mathrm{S}}\right)-7$
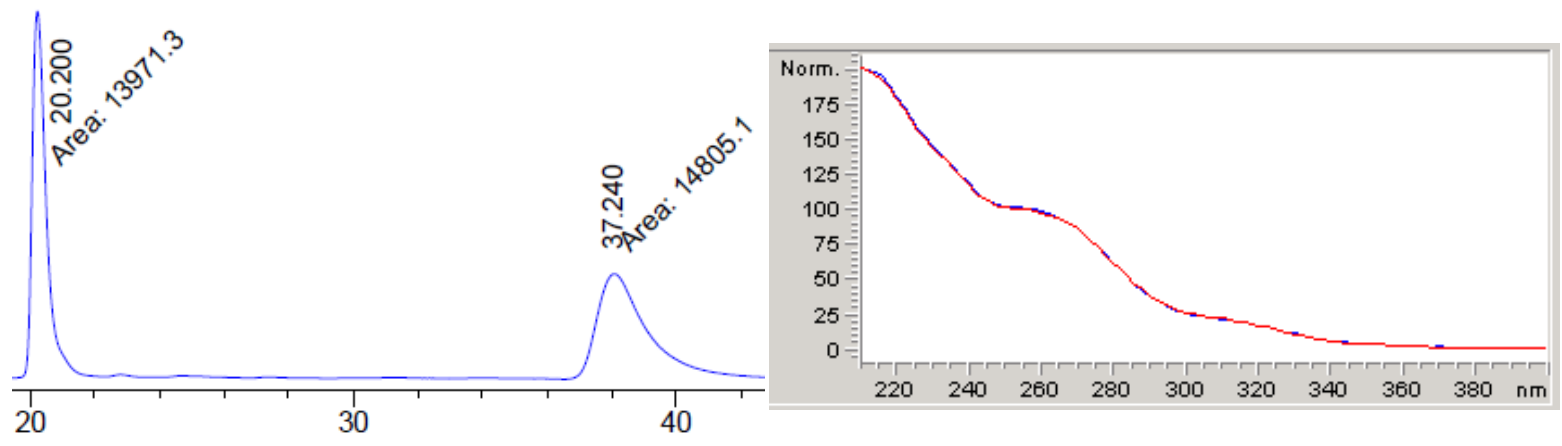

HPLC chromatogram of $\left(P, R_{P}, R\right)-7$
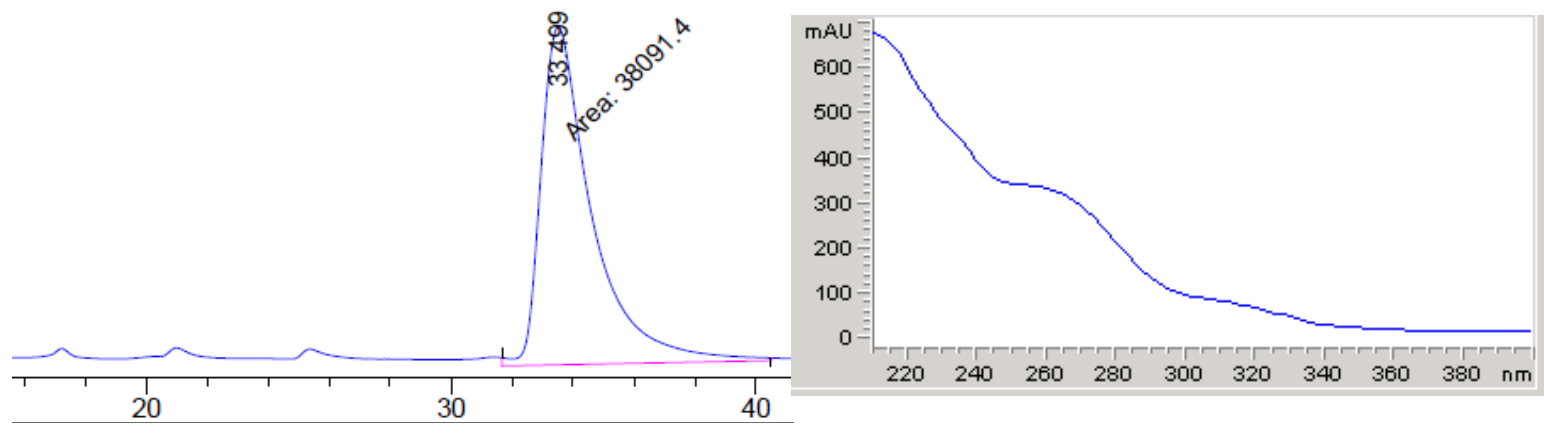

HPLC chromatogram of $\left(M, S_{P}, S\right)-7$
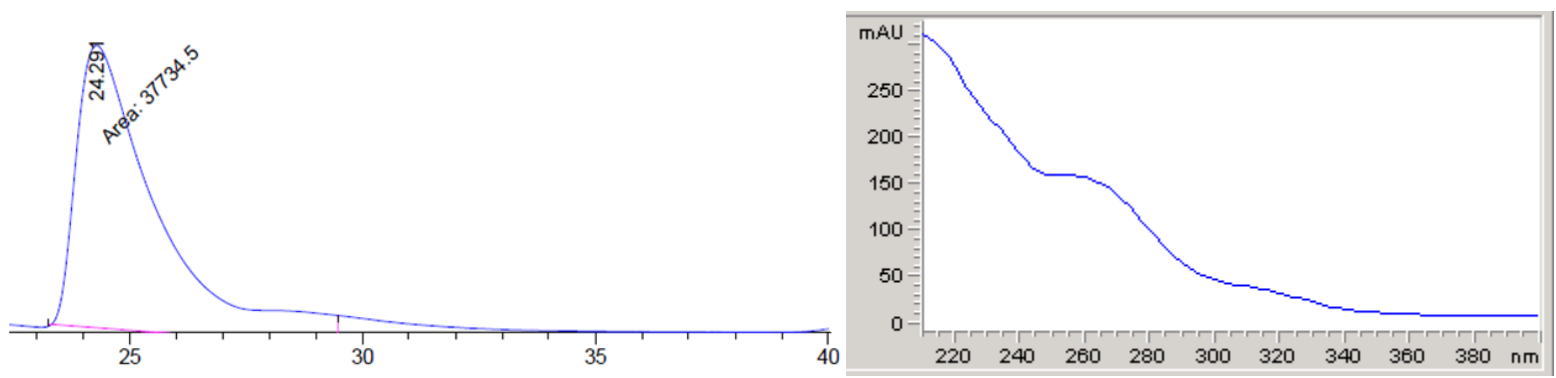


\section{$\underline{\text { X-Ray Crystallography for rac-5 }}$}

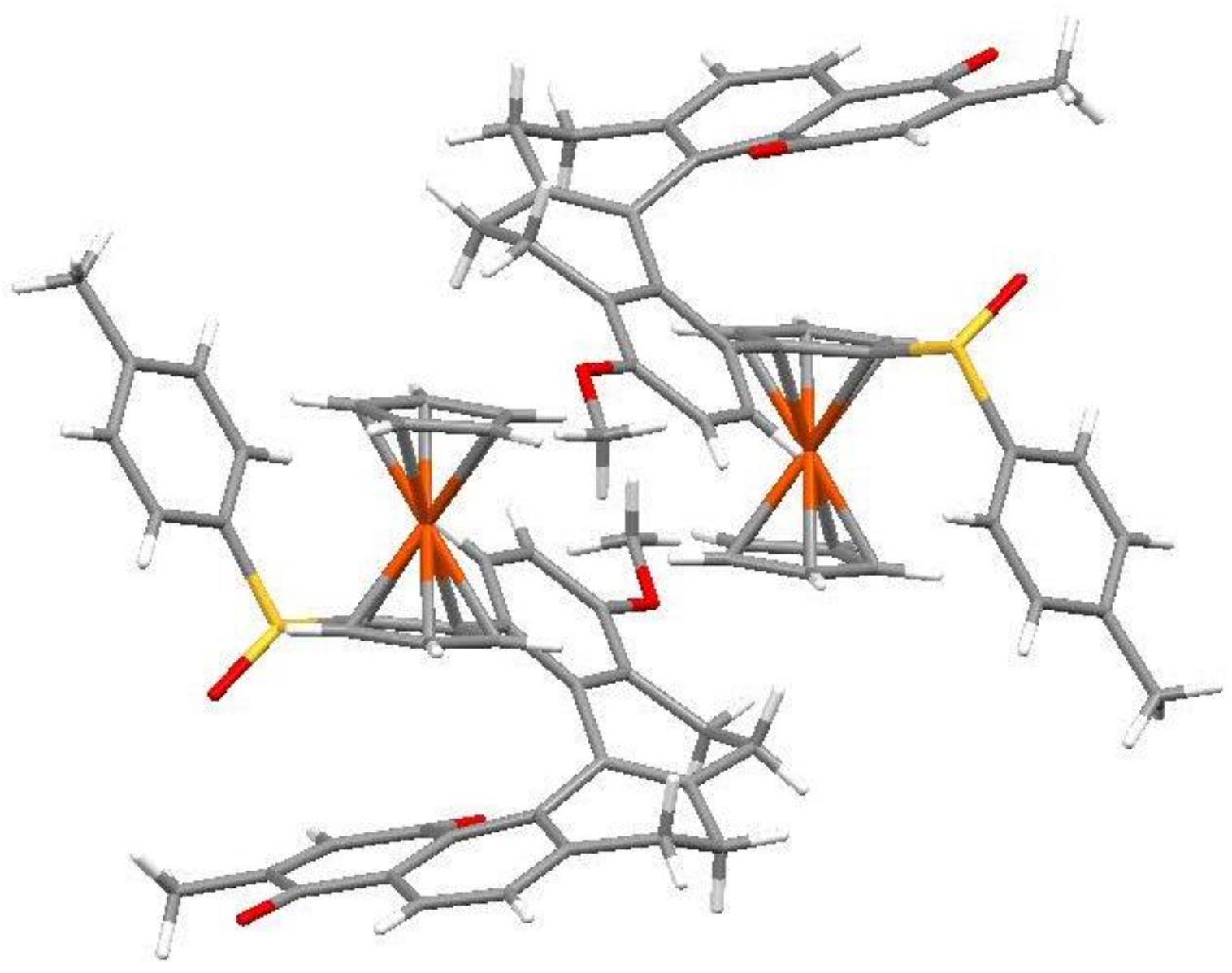


Table 1. Crystal data and structure refinement for rac-5.

\begin{tabular}{|c|c|c|}
\hline Identification code & \multicolumn{2}{|l|}{ datos_Om } \\
\hline Empirical formula & \multicolumn{2}{|l|}{$\mathrm{C} 41 \mathrm{H} 34 \mathrm{Fe} \mathrm{O} 4 \mathrm{~S}$} \\
\hline Formula weight & \multicolumn{2}{|l|}{678.59} \\
\hline Temperature & \multicolumn{2}{|l|}{$296(2) \mathrm{K}$} \\
\hline Wavelength & \multicolumn{2}{|l|}{$0.71073 \AA$} \\
\hline Crystal system & \multicolumn{2}{|l|}{ triclinic } \\
\hline Space group & \multicolumn{2}{|l|}{$P-1$} \\
\hline \multirow[t]{3}{*}{ Unit cell dimensions } & $a=9.5101(2) \AA$ & $\alpha=111.2830(10)^{\circ}$ \\
\hline & $b=12.7112(3) \AA$ & $\beta=95.4860(10)^{\circ}$ \\
\hline & $c=15.1706(3) \AA$ & $\gamma=107.5790(10)^{\circ}$ \\
\hline Volume & \multicolumn{2}{|l|}{$1584.95(6) \AA^{3}$} \\
\hline Z & \multicolumn{2}{|l|}{2} \\
\hline Density (calculated) & \multicolumn{2}{|l|}{$1.422 \mathrm{Mg} / \mathrm{m}^{3}$} \\
\hline Absorption coefficient & \multicolumn{2}{|l|}{$0.586 \mathrm{~mm}^{-1}$} \\
\hline$F(000)$ & \multicolumn{2}{|c|}{708} \\
\hline Crystal size & \multicolumn{2}{|c|}{$0.32 \times 0.21 \times 0.20 \mathrm{~mm}^{3}$} \\
\hline Theta range for data collection: & \multicolumn{2}{|l|}{1.48 to $30.69^{\circ}$. } \\
\hline Index ranges & \multicolumn{2}{|c|}{$-13<=h<=13,-17<=k<=17,-21<=\mid<=21$} \\
\hline Reflections collected & \multicolumn{2}{|l|}{44616} \\
\hline Independent reflections & \multicolumn{2}{|c|}{$9617[\mathrm{R}($ int $)=0.0374]$} \\
\hline Completeness to theta $=30.69^{\circ}$ & \multicolumn{2}{|c|}{$97.8 \%$} \\
\hline Max. and min. transmission & \multicolumn{2}{|l|}{0.8918 and 0.8346} \\
\hline Refinement method & \multicolumn{2}{|c|}{ Full-matrix least-squares on $\mathrm{F}^{2}$} \\
\hline Data / restraints / parameters & \multicolumn{2}{|l|}{9617 / 0 / 427} \\
\hline Goodness-of-fit on $F^{2}$ & \multicolumn{2}{|l|}{1.101} \\
\hline Final $R$ indices [I>2sigma $(I)]$ & \multicolumn{2}{|c|}{$\mathrm{R} 1=0.0422, \mathrm{wR} 2=0.1184$} \\
\hline $\mathrm{R}$ indices (all data) & \multicolumn{2}{|c|}{$\mathrm{R} 1=0.0747, w R 2=0.1511$} \\
\hline Largest diff. peak and hole & \multicolumn{2}{|c|}{0.520 and -0.603 e. $\AA^{-3}$} \\
\hline
\end{tabular}




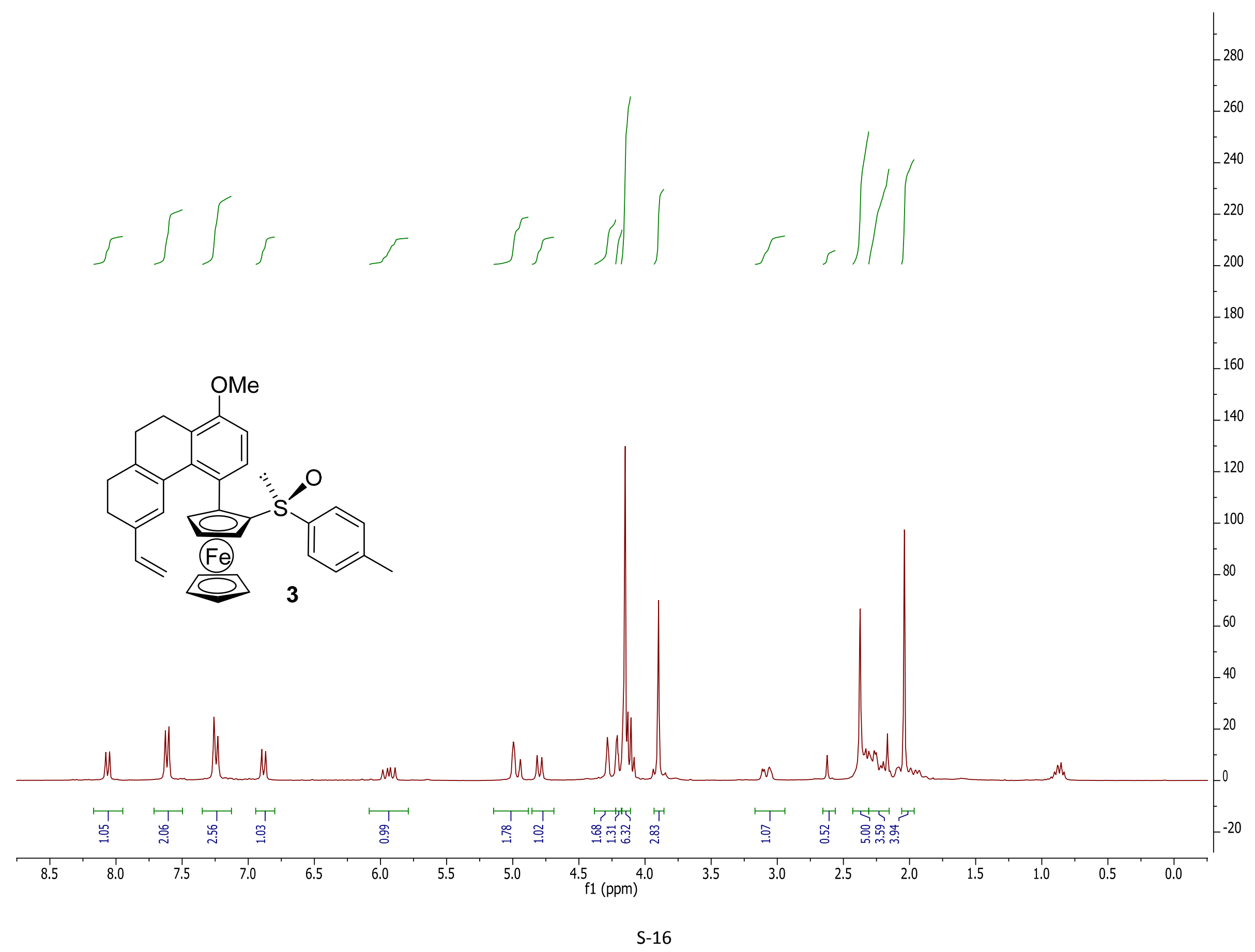




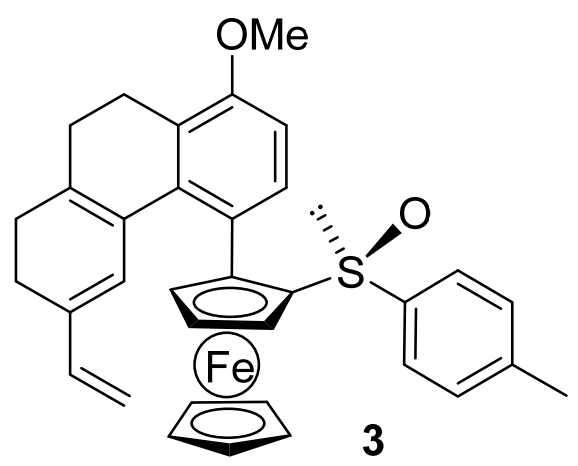




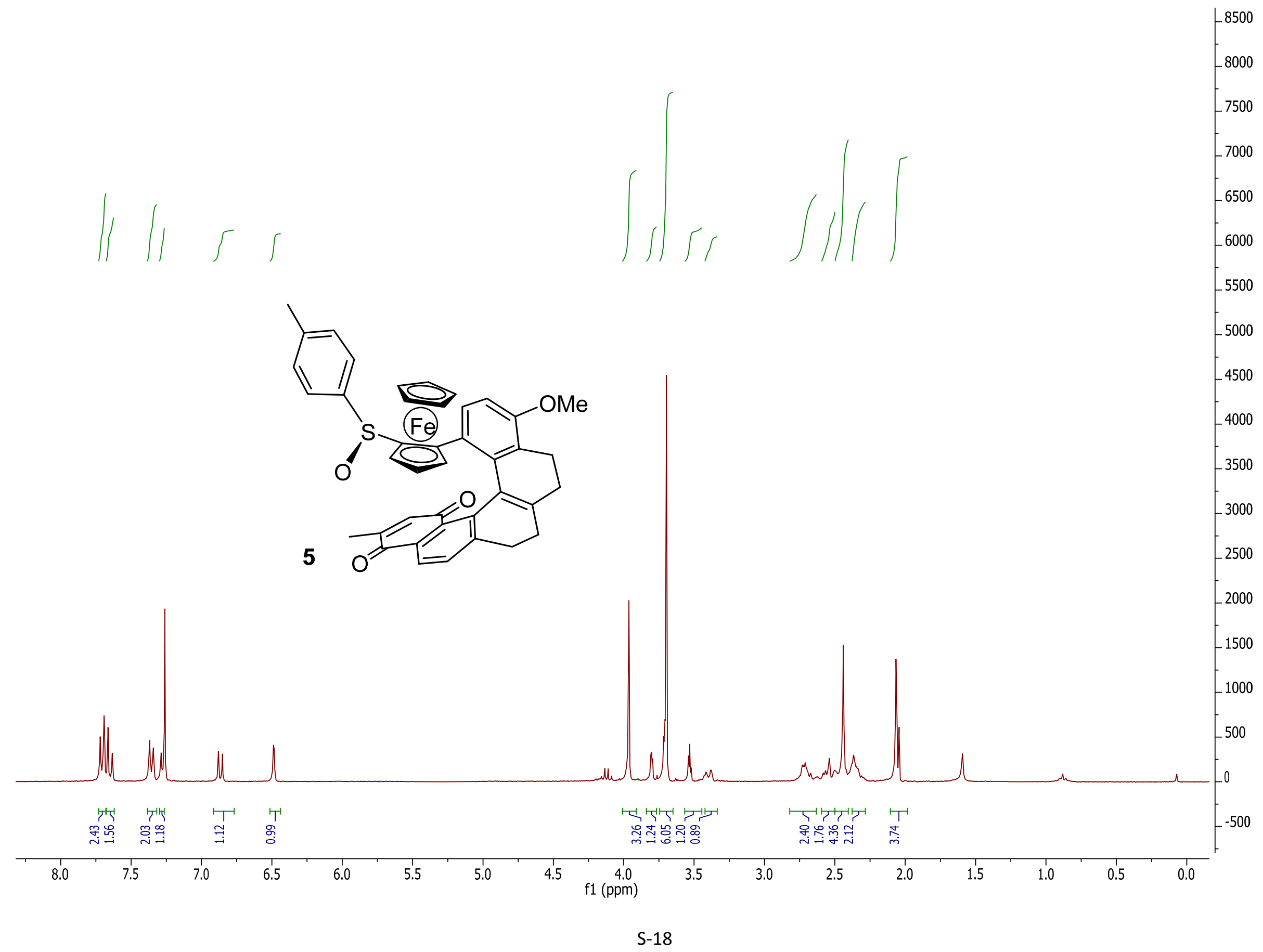




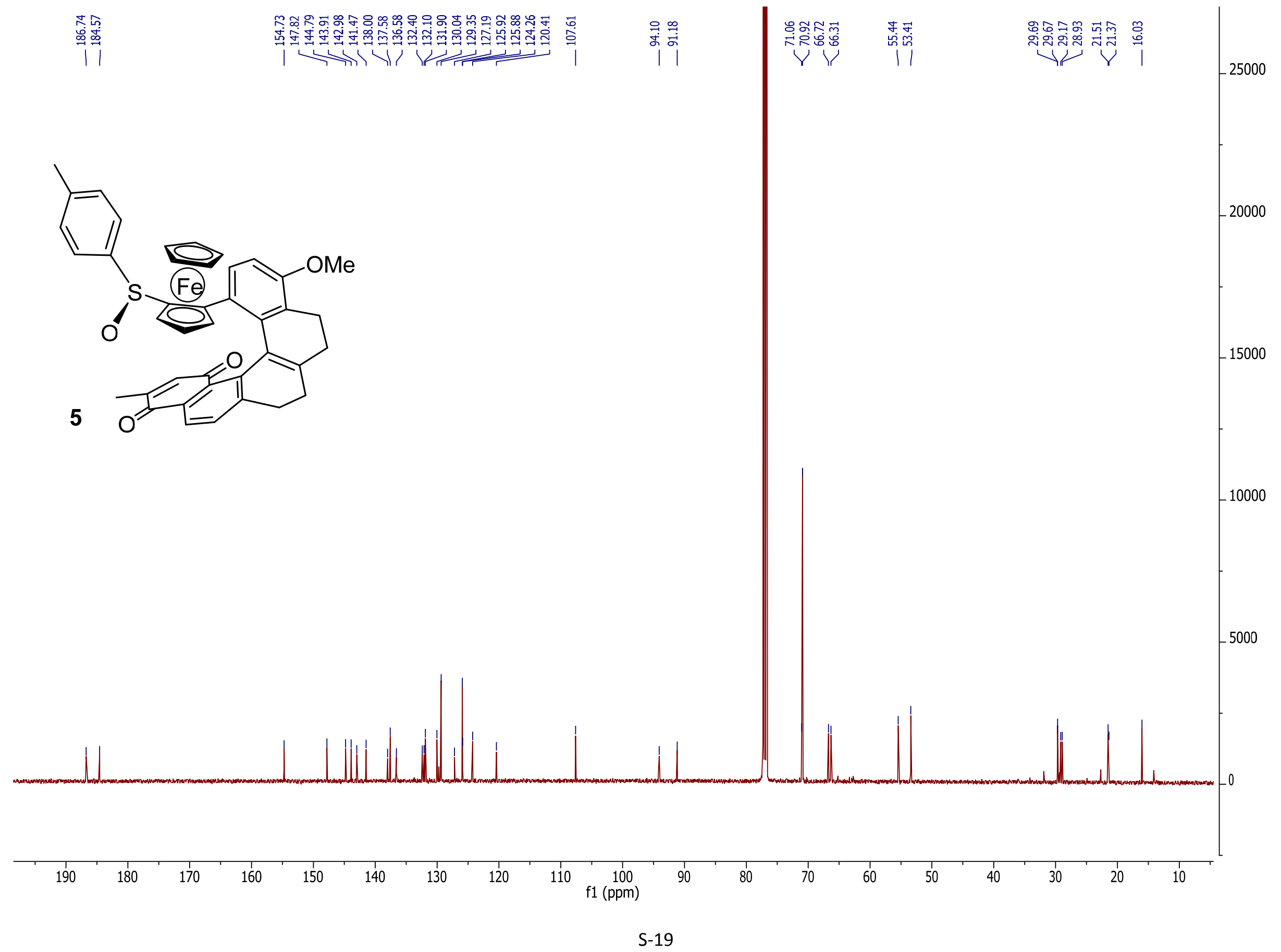




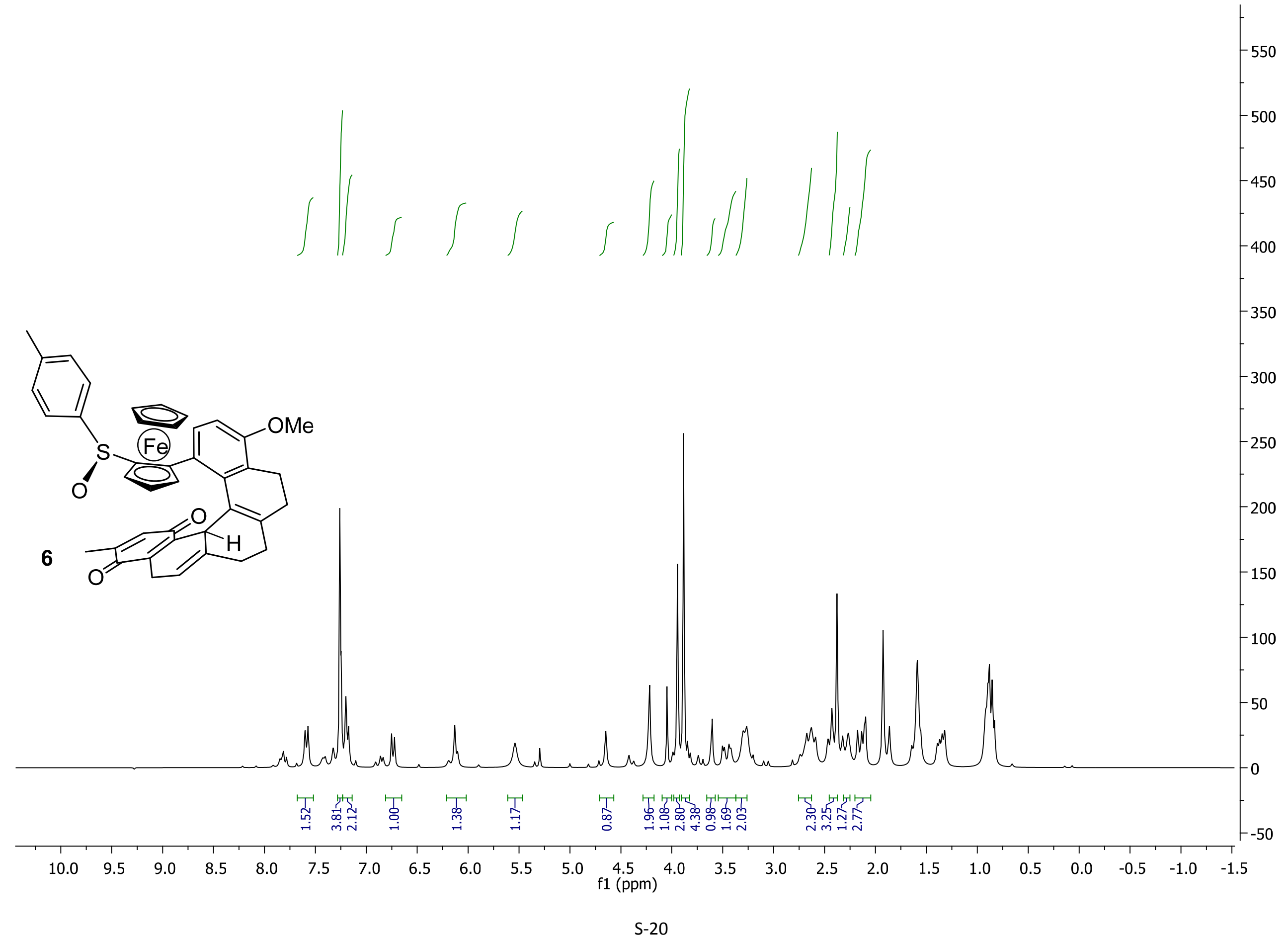




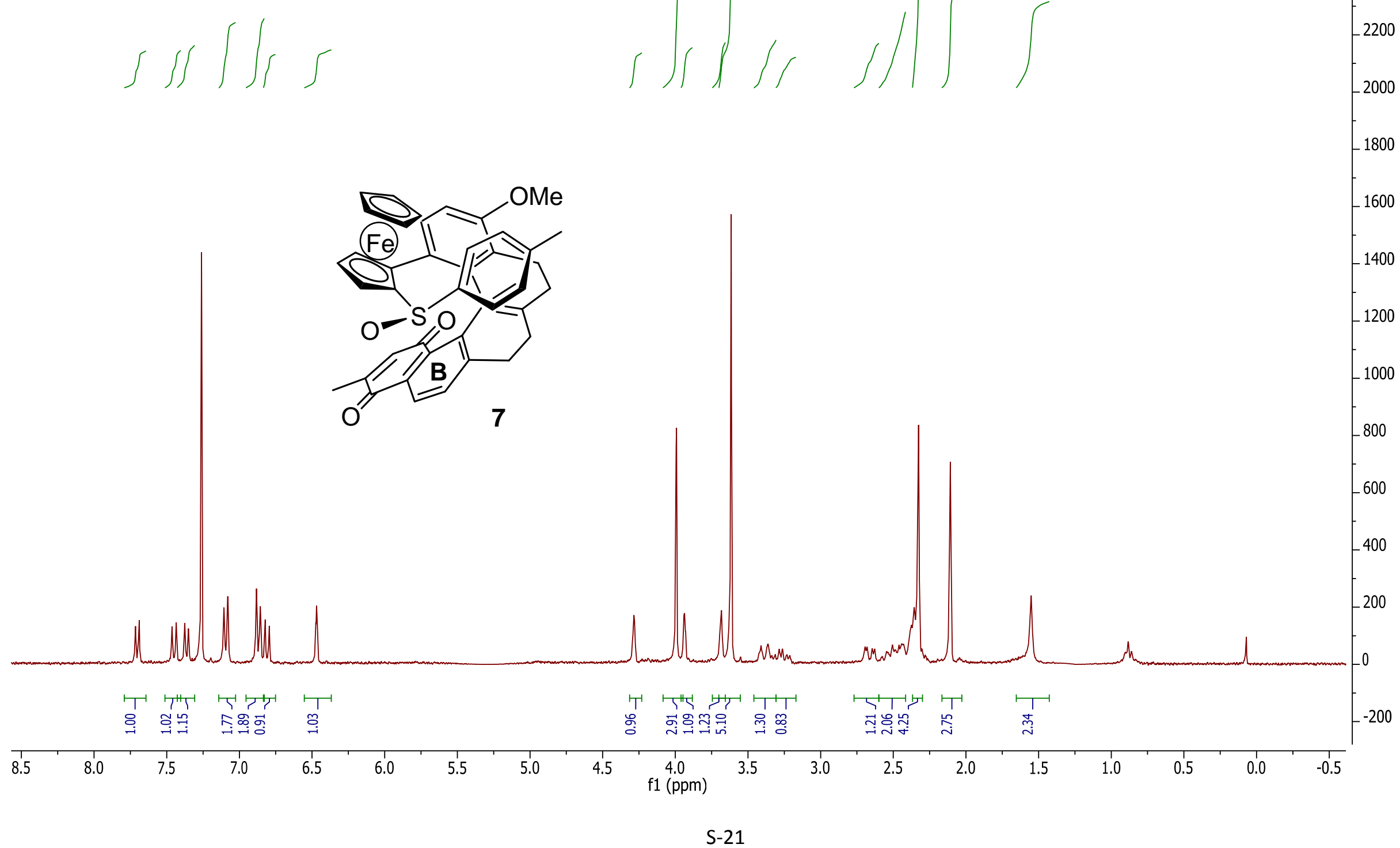




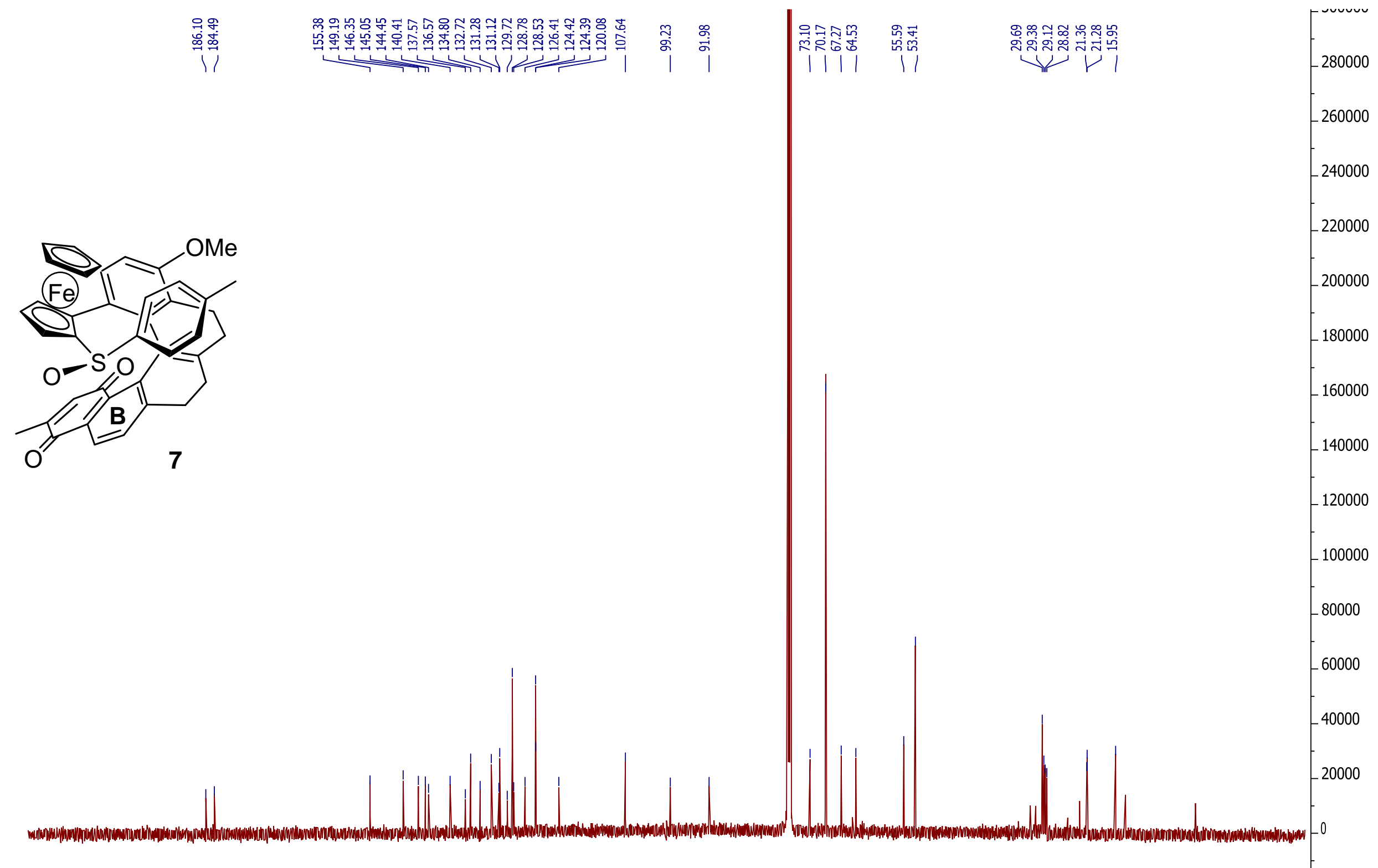




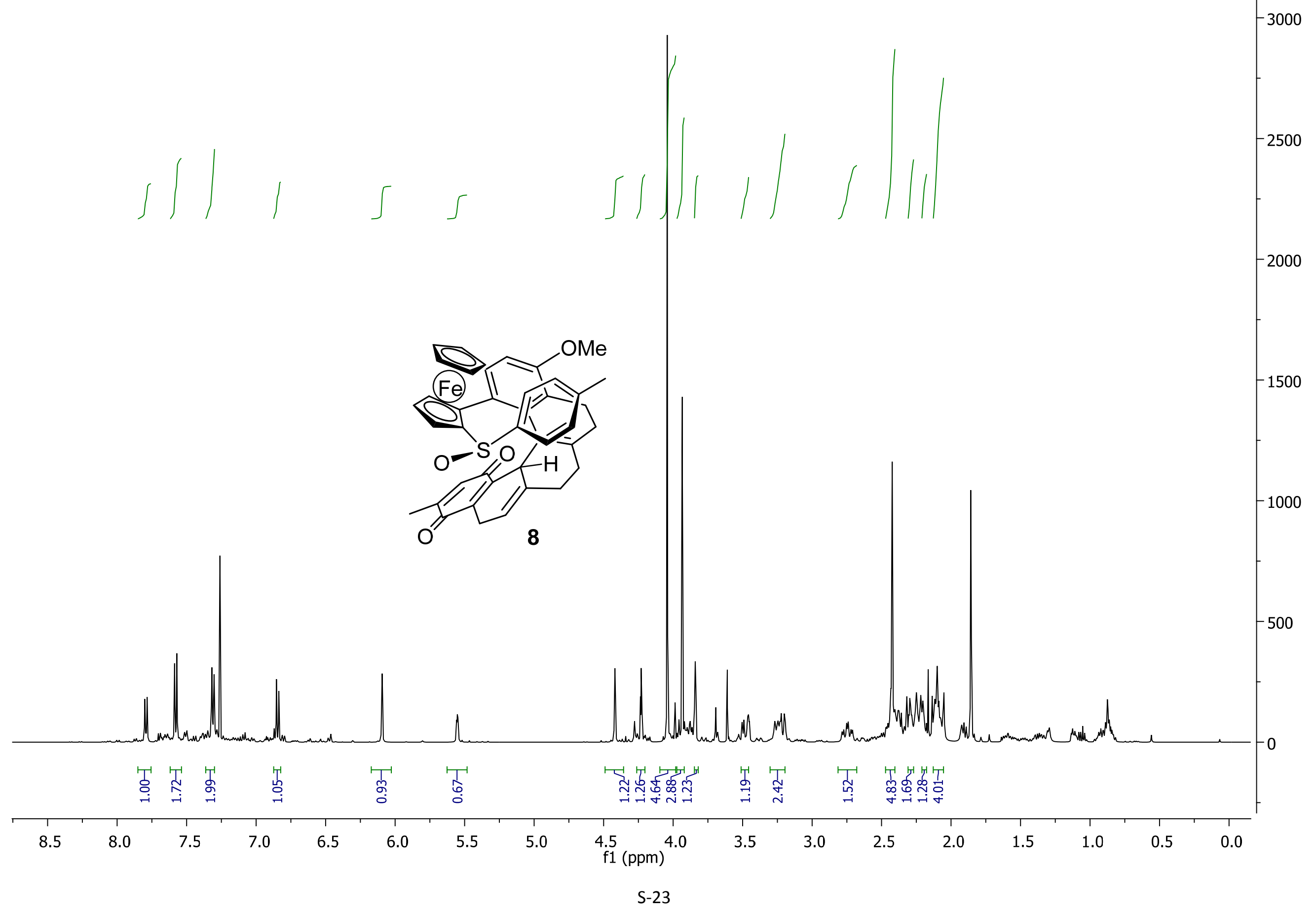

